\title{
Estágios motivacionais para mudança de comportamento em indivíduos que iniciam tratamento para perda de peso
}

\author{
Motivational stages to behavioral change in \\ beginners of weight loss treatment
}

Juliana Medeiros Leão', Letícia Catharina Vitoretti Lisboa', Mariana de Almeida Pereira', Luciane de Freitas Lima', Kelly Cavalheiro Lacerda', Maria Amélia Ribeiro Elias', Aline Silva de Aguiarr', Sheila Cristina Potente Dutra Luquetti

\section{RESUMO}

Objetivo: Embora existam programas efetivos para tratamento da obesidade, são grandes as taxas de abandono. O objetivo deste trabalho foi investigar o estágio motivacional em que se encontravam pacientes com sobrepeso ou obesidade I e II atendidos em ambulatório de Nutrição e seus fatores associados. Métodos: Trata-se de um estudo transversal, com amostragem por conveniência, em que foram coletadas informações da história clínica, antropometria, compulsão alimentar (BES - Binge Eating Scale) e motivação para o tratamento (URICA - University of Rhode Island Change Assessment Scale). Resultados: Dos 48 avaliados, 29,2\% encontravam-se em pré-contemplação, 41,7\%, em contemplação e 29,2\%, em ação. Quem procurou tratamento para controle de alguma comorbidade além da perda de peso teve maior escore de prontidão $(p=0,024)$. Motivação não foi relacionada ao estado nutricional nem à tentativa anterior de perda de peso, mas relacionou-se à orientação profissional anterior ( $p=0,005$ ). Dos $26,8 \%$ que apresentavam sintomatologia moderada ou grave para compulsão alimentar, 90,9\% estavam em contemplação, com diferença significativa quanto à pré-contemplação $(p=0,001)$ e à ação $(p=0,02)$. Conclusão: Esses resultados sugerem que parte dos indivíduos que procuram tratamento para perda de peso não o faz com a motivação necessária e que, se o aconselhamento profissional quanto à importância da perda de

\section{Palavras-chave}

Motivação, perda de peso, obesidade. peso ocorresse antes que patologias associadas à obesidade se instalassem, indivíduos mais motivados poderiam procurar tratamento e evitar tais complicações.

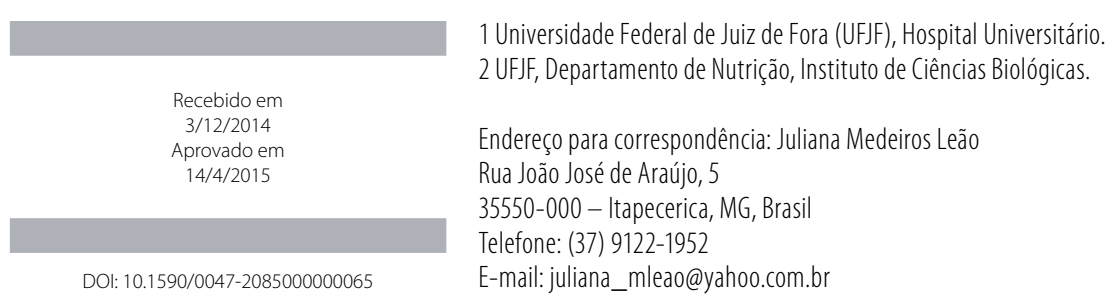




\section{Keywords}

Motivation, weight loss, obesity.

\section{INTRODUÇÃO}

A obesidade é definida como uma condição em que ocorre excessivo acúmulo de gordura no tecido adiposo, a ponto de ser prejudicial à saúde'. No Brasil, entre 2008 e 2009, 49\% da população apresentavam excesso de peso e 14,8\%, obesidade. Tais prevalências aumentaram continuamente ao longo dos 35 anos que separam os inquéritos populacionais nacionais de 1974-1975 e 2008-2009. Na população com mais de 20 anos de idade, o excesso de peso passou de 18,5\% para $50,1 \%$ entre homens e de $28,7 \%$ para $48 \%$ entre mulheres, e a obesidade cresceu de 2,8\% para 12,4\% entre homens e de $8 \%$ para $16,9 \%$ entre mulheres ${ }^{2}$.

As causas do aumento da obesidade ainda não estão esclarecidas. Acredita-se que esteja relacionada ao declínio do gasto energético dos indivíduos, pelo predomínio das ocupações que demandam menor esforço físico e pela redução da atividade física como lazer. Associados a esses fatores, estão a diminuição do consumo de fibras e o aumento do consumo de gorduras e açúcares ${ }^{3}$. Além disso, pesquisas apontam que de $15 \%$ a $22 \%$ dos obesos que procuravam tratamento para emagrecer no Brasil apresentavam transtorno de compulsão alimentar periódica ${ }^{4}$ sem comportamentos compensatórios para perda de peso (DSM-IV) $)^{5}$.

Embora programas de tratamento combinando modificação comportamental, aconselhamento nutricional e atividade física sejam apontados como efetivos no controle da obesidade, taxas de abandono do tratamento variam de 10\% a $80 \%$ em estudos com obesos 6 . Uma das dificuldades para mudanças dietéticas está na crença dos indivíduos de que não há necessidade de alteração dos hábitos alimentares, o que se torna um desafio para os profissionais de saúde em obter sucesso em suas intervenções. Reconhecer a necessidade de alteração dos hábitos alimentares é requisito fundamental para iniciar uma mudança dietética ${ }^{7}$.

De acordo com Modelo Transteórico de Mudança de Comportamento, as pessoas passam por diferentes estágios de motivação durante a modificação de um comportamento-problema. O estágio de mudança no qual a pessoa se encontra possibilita saber o quanto ela está motivada para efetivamente mudar. No estágio de pré-contemplação, a pessoa sequer consegue identificar que tem um problema, dificilmente procura ajuda e, quando o faz, geralmente é incentivada por outro motivo. Na contemplação, já há alguma consciência do problema, mas com alto nível de ambivalência (vantagens e desvantagens de mudar). Na preparação (ou decisão), a marca é o planejamento para a ação. No estágio da ação, o indivíduo está disposto a realizar modificações e aproveita as experiências adquiridas nas tentativas de mudanças realizadas anteriormente. $O$ estágio de manutenção é observado pela estabilização do comportamento em foco, evitando-se a recaída. A avaliação desses estágios é decisiva para a escolha de estratégias terapêuticas mais adequadas e eficazes para o aumento e a manutenção da motivação, favorecendo a mudança efetiva de comportamento ${ }^{8-11}$.

Conhecer melhor os determinantes do comportamento alimentar aumenta as chances de sucesso no tratamento ${ }^{7}$. Embora já esteja bem estabelecida a relação do estilo de vida e do papel da alimentação com o sobrepeso e a obesidade, e já tenham sido realizadas inúmeras pesquisas sobre o tratamento mais adequado para essa enfermidade, nem sempre se consegue alcançar resultados satisfatórios no seu controle, talvez por não abordar o paciente de acordo com o estágio motivacional em que se encontra. Por isso, o objetivo deste trabalho foi investigar o estágio motivacional de pacientes com sobrepeso ou obesidade que procuram tratamento para perda de peso e fatores que interferem na prontidão para mudança de comportamento.

\section{MÉTODOS}

\section{Delineamento do estudo, população, critérios de elegibilidade}

Trata-se de um estudo de delineamento transversal em que se avaliaram adultos e idosos jovens com excesso de peso, em sua primeira consulta no ambulatório de Nutrição Clínica do Hospital Universitário da Universidade Federal de Juiz de Fora (HU/UFJF). Este trabalho utiliza dados preliminares de um projeto maior, que objetiva avaliar o impacto de um 
programa de educação alimentar e nutricional em adultos e idosos com sobrepeso e obesidade grau I ou II.

A amostra deste estudo foi obtida por amostragem de conveniência de acordo com a demanda atendida no ambulatório de Nutrição Clínica do HU/UFJF. A população atendida no ambulatório é encaminhada pelas Unidades de Atenção Básica do município de Juiz de Fora (MG), sendo em média oito encaminhamentos por semana. O ambulatório atende em média 20 pacientes de primeira consulta por mês; desses, cerca de 50\% (10 pacientes) apresentam sobrepeso ou obesidade grau I ou II. As consultas ocorrem às quintas-feiras, das 7 às 12 horas, e os pacientes são atendidos por ordem de chegada.

Foram convidados a participar do estudo todos os indivíduos adultos e idosos jovens, independentemente do sexo, e que apresentavam sobrepeso (IMC $\geq 25 \mathrm{~kg} / \mathrm{m}^{2}$ ), obesidade grau I (IMC $\geq 30$ e $<35 \mathrm{~kg} / \mathrm{m}^{2}$ ) ou obesidade grau II (IMC $\geq 35$ e $<40 \mathrm{~kg} / \mathrm{m}^{2}$ ) em sua primeira consulta no ambulatório. Foram considerados inelegíveis para o estudo os pacientes que apresentaram IMC $<25 \mathrm{~kg} / \mathrm{m}^{2}$ ou $\geq 40 \mathrm{~kg} / \mathrm{m}^{2}$. Os indivíduos não elegíveis ou que se recuram a participar da pesquisa foram atendidos pela nutricionista do ambulatório, conforme rotina já estabelecida. Cinco pacientes foram excluídos por preenchimento incorreto dos questionários.

\section{Aspectos éticos}

O projeto foi aprovado pelo Comitê de Ética em Pesquisa da Universidade Federal de Juiz de Fora no CAAE 11822413.2.0000.5147. Os indivíduos que aceitaram participar da pesquisa assinaram o Termo de Consentimento Livre e Esclarecido.

\section{Procedimentos e coleta dos dados}

Os dados utilizados neste estudo foram coletados antes de qualquer intervenção nutricional no ambulatório. A coleta aconteceu nos meses de março a setembro de 2013, da seguinte maneira:

- Avaliação antropométrica: peso corporal e estatura foram determinados utilizando balança digital e estadiômetro, de acordo com a metodologia de Duarte $^{12}$. $O$ índice de massa corporal, utilizado para classificação do estado nutricional, foi calculado pela fórmula: IMC = peso $(\mathrm{kg}) /$ altura $\left(\mathrm{m}^{2}\right)$, e a classificação do estado nutricional foi realizada conforme proposto pela Organização Mundial de Saúde para adultos $^{13}$. Embora exista uma classificação diferenciada para idosos, neste trabalho eles foram avaliados utilizando a classificação de adultos, uma vez que são, em sua maioria, idosos jovens (entre 60 e 69 anos), tendo apenas um indivíduo na amostra com idade superior a 70 anos.

- Anamnese e dados cadastrais: foram coletadas informações sobre dados cadastrais e demográficos, e história clínica e de tratamentos anteriores.
- Avaliação da compulsão alimentar: utilizou-se a Escala de Compulsão Alimentar Periódica (Binge Eating Scale - BES), questionário autopreenchível, já traduzido para o português e validado, desenvolvido para avaliar a gravidade da compulsão alimentar periódica em indivíduos obesos que procuram tratamento para emagrecer ${ }^{4}$ É uma escala Likert com 16 itens que avaliam as manifestações comportamentais, sentimentos e cognições envolvidos num episódio de compulsão alimentar periódica. Resultado maior ou igual a 27 pontos classifica o indivíduo em compulsão grave, entre 18 e 26 pontos, em moderada e menor ou igual a 17 pontos, em ausência de compulsão.

- Avaliação do estágio motivacional: para avaliar o estágio de motivação, utilizou-se a escala URICA (University of Rhode Island Change Assessment Scale). A URICA investiga os estágios motivacionais de indivíduos em relação à modificação de qualquer tipo de problema comportamental, como meio de guiar abordagens de tratamento adequadas. Neste estudo, foi utilizada a versão adaptada e validada para o comportamento do comer compulsivo ${ }^{14}$. Trata-se de um instrumento autoaplicável, em escala Likert, que contém 24 itens com cinco alternativas de respostas variando de "discordo totalmente" (1 ponto) a "concordo totalmente" (5 pontos). É dividido em quatro subescalas, com seis itens cada: pré-contemplação (questões 1, 5, 7, 16, 19 e 22), contemplação (questões 3, 6, 9, 13, 14 e 17), ação (questões 2, 4, 8, 11, 18 e 23) e manutenção (questões 10, 12, 15, 20, 21 e 24). O escore de prontidão para a mudança é avaliado por meio da equação: média de pontos da contemplação + média de pontos da ação + média de pontos da manutenção - média de pontos da pré-contemplação. Como ponto de corte foi utilizada a proposta de até 8 pontos para pré-contemplação, entre 8 e 10,33 para contemplação e acima de 10,33 para ação ${ }^{14}$.

\section{Análise estatística}

A análise estatística foi feita utilizando o programa SPSS 12.0. Primeiramente, foi realizada análise descritiva das condições avaliadas, com cálculo das frequências, medidas de tendência central e de dispersão. Nos testes de relação, o escore de prontidão, em sua forma contínua, foi a variável dependente, enquanto sexo, motivo do encaminhamento ao ambulatório, tentativa anterior de perda de peso, orientação profissional anterior, compulsão alimentar periódica e classificação do IMC foram as variáveis dependentes, em sua forma categórica. Por não apresentar distribuição-padrão pelo teste de normalidade dos dados de Shapiro-Wilk, a comparação de médias do escore de prontidão entre as categorias das variá- 
veis independentes foi obtida por meio do teste de U-Mann-Whitney. Para comparação entre os estágios de motivação e a presença de compulsão alimentar periódica, foi utilizado teste exato de Fisher. Em todas as análises foi considerado o nível de significância de 0,05.

\section{RESULTADOS}

Foram avaliados 48 indivíduos, dos quais $41(85,4 \%)$ eram do sexo feminino e $7(14,6 \%)$ eram do sexo masculino, com média de idade de 47,7 $\pm 12,6$ anos, sendo a mínima de 20 anos e a máxima de 72 anos.

A tabela 1 apresenta a classificação dos sujeitos quanto ao estado nutricional, compulsão alimentar periódica e estágio motivacional para mudança de comportamento. A maioria dos sujeitos se encontrava em contemplação pela URICA e não apresentaram compulsão.

Tabela 1. Estado nutricional, compulsão alimentar periódica e estágio de motivação para o tratamento entre indivíduos com excesso de peso atendidos no Ambulatório de Nutrição Clínica, em 2013

\begin{tabular}{lc}
\hline Categoria & Frequência n $(\%)$ \\
\hline Estado nutricional & $17(35,4)$ \\
Sobrepeso & $18(37,5)$ \\
Obesidade grau I & $13(27,1)$ \\
Obesidade grau II & $48(100,0)$ \\
& \\
Compulsão alimentar periódica & $30(73,2)$ \\
Ausente & $8(19,5)$ \\
Moderada & $3(7,3)$ \\
Grave & $41(100,0)^{*}$ \\
& \\
Motivação para mudança de comportamento & $14(29,2)$ \\
Pré-contemplação & $20(41,7)$ \\
Contemplação & $14(29,2)$ \\
Ação & $48(100,0)$ \\
\hline
\end{tabular}

* Sete sujeitos foram excluídos das análises de compulsão alimentar por preenchimento inadequado do questionário BES.

Os indivíduos foram questionados quanto ao motivo do encaminhamento ao ambulatório, sendo as respostas agrupadas em "perda de peso" e "patologia associada à perda de peso", ou seja, quando desejavam, além de perder peso, controlar alguma patologia associada à obesidade (hipertensão, diabetes, dislipidemia, problemas articulares). Apenas um indivíduo não se enquadrou nessas duas categorias, respondendo que procurou o ambulatório para receber orientações quanto à prática de exercícios físicos. A maioria dos entrevistados (62,5\%) procurou o ambulatório para perda de peso, já havia recebido orientação profissional (56,2\%) e tentou perder peso anteriormente por meio de diversas estratégias (77,1\%) (Tabela 2).

Tabela 2. Motivo do encaminhamento e estratégias anteriores ao tratamento de indivíduos com excesso de peso atendidos no Ambulatório de Nutrição Clínica, em 2013

\begin{tabular}{lc}
\hline \multicolumn{1}{c}{ Categoria } & Frequência n $(\%)$ \\
\hline Motivo do encaminhamento ao ambulatório & $30(62,5)$ \\
Perda de peso & $17(35,4)$ \\
Patologias associadas à perda de peso & $1(2,1)$ \\
Outro motivo & $48(100,0)$ \\
& \\
Orientação anterior ao tratamento atual & $21(43,8)$ \\
Não & $15(31,2)$ \\
De médico & $10(20,8)$ \\
De nutricionista & $2(4,2)$ \\
De médico e de nutricionista & $48(100,0)$ \\
& \\
Estratégias anteriores para perda de peso & $11(22,9)$ \\
Nenhuma & $12(25,0)$ \\
Dieta por conta própria & $11(22,9)$ \\
Dieta orientada por médico & $9(18,8)$ \\
Dieta orientada por nutricionista & $4(8,3)$ \\
Dieta de revista & $15(31,3)$ \\
Remédios para emagrecer & $4(8,3)$ \\
Exercícios físicos & $48(100,0)^{*}$ \\
\hline
\end{tabular}

* Soma dos valores ultrapassa 100,0\% em virtude da possibilidade de escolha de mais de uma resposta.

O escore de prontidão variou de 4,2 a 13,3 pontos, com média de 9,2 $\pm 2,1$. Não houve diferença estatística entre as médias de escore de prontidão segundo o sexo. Procurar o ambulatório por motivo de patologia relacionada à obesidade, além de exclusivamente para perda de peso, foi associada a maior escore de prontidão $(p=0,024)$. Não houve diferença na média de escore de prontidão entre indivíduos que tentaram perder peso anteriormente e os que nunca tentaram, nem quando analisada cada estratégia separadamente (dados não demonstrados). Entretanto, aqueles que já receberam alguma orientação profissional tiveram um escore de prontidão maior que aqueles que nunca receberam ( $p=0,005)$ (Tabela 3). Não houve diferença do escore de prontidão entre as três categorias de estado nutricional. A presença de compulsão alimentar periódica não alterou significativamente a média do escore de prontidão, porém foi associada ao estágio motivacional de contemplação (Tabela 4) quando comparada à pré-contemplação $(p=0,001)$ e à ação $(p=0,02)$ pelo teste exato de Fisher. 
Tabela 3. Análises de relação entre as variáveis e o resultado do Escore de Prontidão obtido pela URICA

\begin{tabular}{|c|c|c|c|}
\hline & $\mathrm{n}$ & Média \pm desvio-padrão & $\mathrm{p}$ \\
\hline \multicolumn{4}{|l|}{ Sexo } \\
\hline Feminino & 41 & $9,10 \pm 2,04$ & 0,650 \\
\hline Masculino & 7 & $9,64 \pm 2,77$ & \\
\hline \multicolumn{4}{|c|}{ Motivo do encaminhamento ao ambulatório ${ }^{1}$} \\
\hline Perda de peso & 30 & $8,66 \pm 1,98$ & $0,024^{*}$ \\
\hline Doença relacionada à obesidade & 17 & $10,21 \pm 2,13$ & \\
\hline \multicolumn{4}{|l|}{ Tentativa anterior de perda de peso } \\
\hline Não & 11 & $8,59 \pm 2,75$ & 0,439 \\
\hline $\operatorname{sim}$ & 37 & $9,36 \pm 1,93$ & \\
\hline \multicolumn{4}{|l|}{ Orientação profissional anterior } \\
\hline Não & 21 & $8,18 \pm 2,10$ & $0,005^{*}$ \\
\hline $\operatorname{sim}$ & 27 & $9,96 \pm 1,85$ & \\
\hline \multicolumn{4}{|l|}{ Compulsão alimentar periódica } \\
\hline Não & 30 & $8,83 \pm 2,37$ & 0,479 \\
\hline $\operatorname{Sim}$ & 11 & $9,57 \pm 0,80$ & \\
\hline \multicolumn{4}{|l|}{ IMC } \\
\hline Sobrepeso & 17 & $9,46 \pm 2,09$ & $0,261^{2}$ \\
\hline Obesidade I & 18 & $8,43 \pm 2,36$ & $0,078^{3}$ \\
\hline Obesidade II & 13 & $9,87 \pm 1,62$ & $0,645^{4}$ \\
\hline
\end{tabular}

* Diferença significativa - teste U-Mann-Whitney $(p<0,05) .{ }^{1}$ Nesta análise foi excluído um indivíduo, cuja resposta não se enquadrava nas duas opções. ${ }^{2}$ Comparação entre sobrepeso e obeso $l_{;}{ }^{3}$ Comparação entre obeso 1 ell; ${ }^{4}$ Comparação entre sobrepeso e obeso ll.

Tabela 4. Análise de associação entre os estados motivacionais para mudança de comportamento e a presença de compulsão alimentar periódica

\begin{tabular}{lccc}
\hline \multirow{2}{*}{ Estágios motivacionais } & \multicolumn{2}{c}{ Compulsão alimentar periódica } & \\
\cline { 2 - 3 } & $\begin{array}{c}\text { Ausência } \\
\mathbf{n}(\%)\end{array}$ & $\begin{array}{c}\text { Grau leve ou grave } \\
\mathbf{n}(\%)\end{array}$ & $\mathbf{p}^{*}$ \\
\hline Contemplação & $7(23,3)$ & $10(90,9)$ & \\
Pré-contemplação & $13(43,3)$ & $0(0,0)$ & $0,001^{*}$ \\
Ação & $10(33,3)$ & $1(9,1)$ & $0,02^{*}$ \\
Total & $30(100,0)$ & $11(100,0)$ & \\
\hline
\end{tabular}

* Diferença significativa pelo Teste Exato de Fisher, em relação ao estágio de Contemplação.

\section{DISCUSSÃO}

No presente estudo, foi predominante a participação de muIheres que procuram tratamento para perda de peso, o que já era esperado, uma vez que reflete o perfil de atendimento no ambulatório em estudo. Essa predominância feminina também foi observada por outros autores, que a associam à maior preocupação com estética e saúde pelas mulheres em relação aos homens ${ }^{15,16}$.

A prontidão para mudança pode ser conceituada como a importância que o sujeito dá ao seu problema, combinada com a sua capacidade de mudar 9 . A maioria dos participantes deste estudo $(41,7 \%)$ estava no estágio de contemplação, indicando que reconhecem o problema, estão dispostos a superá-lo, mas ainda não apresentam um comprometimento decisivo. Nesse estágio, há conhecimento dos benefícios da mudança (como a adoção de hábitos para perda de peso), mas diversas barreiras impedem a ação desejada (por exemplo, preço, falta de tempo, sabor desagradável de alimentos tidos como saudáveis) $)^{7}$. Dos demais indivíduos, 29,2\% se encontravam em ação, estágio esperado em que estejam os indivíduos que procuram tratamento, enquanto outros $29,2 \%$ se encontravam em pré-contemplação, ainda sem a motivação necessária para iniciar uma mudança de comportamento.

A comparação dos resultados com a literatura torna-se difícil por causa das diferenças metodológicas entre os estudos. O primeiro estudo de validação de uma versão brasileira da URICA para mudanças de hábitos alimentares foi publicado no final de 201211, talvez, por isso, existam poucos estudos brasileiros utilizando esse método para avaliar mudança de comportamento entre obesos. Além disso, os pontos de corte propostos por Bittencourt ${ }^{14}$ não incluem os estágios de preparação e manutenção, dificultando a comparação com outros métodos de avaliação dos estágios. Estudo americano utilizando a versão não específica da URICA encontrou a maioria dos obesos $(46,7 \%)$ em ação para mudança de comportamento relacionada à perda de peso ${ }^{17}$. Estudos com outras metodologias de determinação dos estágios de mudança identificaram a maioria dos indivíduos com excesso de peso em estágio de ação ${ }^{18,19}$.

Embora a procura por tratamento seja uma característica do estágio de açãoª a maioria dos indivíduos deste estudo se encontrava em pré-contemplação. Para atendimento nutricional no ambulatório em que se desenvolveu este estudo, os indivíduos necessitavam ser encaminhados por um médico da atenção básica. A orientação médica para o controle de uma doença é considerada uma motivação extrínseca ao tratamento, bem como as queixas de familiares sobre o consumo alimentar. A pressão de outras pessoas leva indivíduos que ainda não estão em estágio de ação a procurarem tratamento. Diferente da motivação intrínseca, que surge do próprio indivíduo, a extrínseca pode atuar de forma positiva ou negativa nas mudanças no comportamento alimentar ${ }^{7,8}$. Não houve diferença do escore de prontidão em relação ao estado nutricional. Uma vez que este estudo somente considerou indivíduos com sobrepeso, obesidade graus I e II, a comparação com outros estudos é limitada. Sbrocco et al. também não encontraram relação entre motivação e estado nutricional ${ }^{19}$. Por outro lado, obesos atendidos na atenção primária, em estágios mais avançados de motivação, tinham menor $\mathrm{IMC}^{18}$.

Quanto ao motivo do encaminhamento ao ambulatório, 35,4\% relataram, além do emagrecimento, a necessidade de controlar alguma comorbidade relacionada à obesidade. Estes apresentaram média de escore de prontidão 1,55 pontos 
acima dos que desejavam apenas perder peso $(p=0,024)$. No trabalho de Koehnlein et al. ${ }^{15}$, uma maior porcentagem de indivíduos (60\%) procurou atendimento por "saúde", associada ou não à "estética", e aqueles com doenças associadas e risco para doenças cardiovasculares e metabólicas perderam mais peso durante o período analisado, sugerindo uma influência positiva de dois fatores, estética e saúde, na motivação do paciente pela busca do atendimento nutricional.

No estudo de Koehnlein et al. ${ }^{15}, 75 \%$ dos pacientes de uma clínica particular não seguiram recomendações médicas focadas na restrição alimentar e 95\% dos obesos que iniciaram uma dieta para perda de peso fracassaram em sua manutenção. Neste trabalho, apenas 22,9\% da amostra nunca haviam utilizado alguma estratégia para perda de peso. Os demais haviam utilizado uma ou várias estratégias, como dietas, uso de medicamentos e prática de exercícios físicos. Não houve diferença na média do escore de prontidão entre os indivíduos que já tentaram perder de peso e aqueles que estavam na primeira tentativa. Ainda no estudo de Koehnlein et $a . .^{15}$, maior perda de peso foi relacionada ao fato de não ter realizado dieta anteriormente, mas, segundo a revisão de Teixeira et al..$^{20}$, menos tentativas anteriores foi um dos mais consistentes preditores de perda de peso entre os estudos.

Ao serem questionados sobre orientação profissional anterior, 31,2\% relataram já terem sido orientados por médico, 20,8\%, por nutricionista e 4,2\%, por ambos os profissionais. Esses pacientes anteriormente orientados apresentaram uma média de escore de prontidão 1,78 ponto acima dos que nunca haviam recebido orientação $(p=0,005)$. Isso sugere que, apesar de a orientação anterior não ter sido efetiva no tratamento, ou ter surtido efeito temporário, ela foi um fator motivador para a mudança atual do comportamento. Chimenti et al. ${ }^{21}$ evidenciaram que a orientação não é suficiente para o cumprimento das recomendações dietéticas e que a presença de tratamentos anteriores configura o comportamento de peso flutuante. Contudo, no estudo de Wee et $a l .{ }^{18}$, a orientação médica prévia foi fortemente associada à percepção do excesso de peso como um risco à saúde, e tal percepção foi associada aos maiores escores de prontidão para perda de peso. Por isso, acreditam que os profissionais de saúde podem desempenhar um papel importante na motivação de pacientes, ajudando-os na percepção de risco para a saúde relacionado ao excesso de peso.

Nesta amostra, 26,8\% dos indivíduos apresentaram sintomatologia leve ou grave de compulsão alimentar periódica. De acordo com Freitas et al. ${ }^{4}$, cerca de $30 \%$ dos pacientes obesos que procuram tratamentos para emagrecer são portadores do transtorno da compulsão alimentar periódica. A prevalência em programas para perda de peso latino-americanos varia de $16 \%$ a $51,6 \%{ }^{22}$. Poucos estudos avaliam estágio de prontidão para mudança em indivíduos obesos com compulsão alimentar. Neste estudo, 90,9\% dos compulsivos estavam em contemplação, com diferença significativa em relação aos outros estágios, enquanto Moraes e Oliveira ${ }^{16}$ encontraram que $93,3 \%$ das mulheres com obesidade moderada, em tratamento para compulsão alimentar, estavam em estágio de ação pelo método da régua de prontidão.

De acordo com Teixeira et al. ${ }^{20}$, episódios de compulsão alimentar pré-tratamento foram um mau preditor de perda de peso entre os estudos. Entre os estudos analisados em revisão de literatura realizada pelos autores, pacientes com compulsão alimentar periódica apresentam distorções no pensamento e na percepção, que interferem no tratamento. Tendem a privilegiar a satisfação imediata, ignorando as suas consequências, e estar mais suscetíveis a sobrecargas emocionais que impulsionam ao episódio do comer compulsivo e dificultam a manutenção da perda de peso a longo praz० ${ }^{23,24}$. A ambivalência própria do estágio de contemplação pode estar associada à compulsão alimentar por esse motivo, uma vez que se tornar ou permanecer saudável envolve metas a longo prazo, enquanto um episódio compulsivo satisfaz uma meta a curto prazo de prazer ${ }^{25}$.

Este trabalho apresenta limitações próprias de um estudo transversal, que não permite identificar relações de causa e efeito e também se limita pela técnica de amostragem por conveniência, o que faz com que esses resultados possam não ser representativos da população. Por outro lado, todos os indivíduos elegíveis ao estudo foram convidados a participar.

Saber se existe um momento ideal para que os indivíduos que querem perder peso se envolvam em programas para emagrecimento é um objetivo antigo quando o assunto é o tratamento da obesidade, mas raramente tem sido praticado. Avaliar as características dos participantes e o nível de prontidão ao entrarem em um programa de redução de peso permite otimizar a eficácia da intervenção com melhor adequação do tratamento às características dos participantes ${ }^{20}$. A maioria dos programas de tratamento considera, em geral, que os indivíduos estão prontos para a ação, o que tem se mostrado insustentável em muitas situações ${ }^{25}$. Segundo Cochrane ${ }^{26}$, alguns pacientes com excesso de peso têm pouco ou nenhum interesse em emagrecer. Parte dos que procuram ajuda reluta em aceitar sua responsabilidade em sua própria perda de peso. Outros, no entanto, estão dispostos a aprender como eles podem assumir o controle de sua própria saúde e vão valorizar as informações oferecidas.

Um dos papéis do nutricionista é o de ajudar as pessoas a modificarem seus hábitos alimentares, por meio da assistência nutricional a indivíduos e grupos populacionais. Porém, assistência é mais do que fornecer uma informação. O conhecimento não leva à mudança, mas funciona como um instrumento quando as pessoas desejam mudar. A maioria dos pacientes com excesso de peso já tentou muitas dietas, portanto é bem informada sobre nutrição. A relação de confiança com o paciente dá ao profissional uma oportunidade para corrigir equívocos da perda de peso ${ }^{25,26}$. 
Avaliar a motivação para mudança é importante para nortear as estratégias que serão utilizadas para cada paciente. Os estágios de mudança proporcionam um grande desafio para o desenvolvimento da intervenção. A intensidade, a duração e o tipo de intervenção devem ser sensíveis ao estágio de mudança do indivíduo ${ }^{9}$.

\section{CONCLUSÕES}

Esses resultados sugerem que parte dos indivíduos que procuram tratamento para perda de peso não o faz com a motivação necessária e que o aconselhamento profissional é capaz de aumentar tal motivação e, consequentemente, as chances de sucesso. Sugerem também que esses indivíduos, muitas vezes, só alcançam maior estágio de motivação ao apresentar sintomas de comorbidades associadas. Talvez, se o aconselhamento profissional quanto à importância da perda de peso ocorresse antes que patologias associadas à obesidade se instalassem, indivíduos mais motivados poderiam procurar tratamento e evitar tais complicações. Ainda que alguns indivíduos com excesso de peso não estejam prontos para o tratamento, cabe aos profissionais de saúde o aconselhamento, baseado não somente em informações de saúde, mas também em estratégias que visem à motivação para mudança de comportamento.

\section{CONTRIBUIÇÕES INDIVIDUAIS}

Juliana Medeiros Leão - Participou da elaboração do projeto, desenvolvimento do trabalho, análise e interpretação dos dados, e da escrita deste artigo.

Letícia Catharina Vitoretti Lisboa - Participou da elaboração do projeto, desenvolvimento do trabalho e da revisão e aprovação da versão final deste artigo.

Mariana de Almeida Pereira, Luciane de Freitas Lima e Kelly Cavalheiro Lacerda - Participaram do desenvolvimento do trabalho e da revisão e aprovação da versão final deste artigo.

Maria Amélia Ribeiro Elias - Participou da elaboração do projeto, desenvolvimento do trabalho e da revisão e aprovação da versão final deste artigo.

Aline Silva de Aguiar e Sheila Cristina Potente Dutra Luquetti - Participaram da elaboração do projeto, desenvolvimento do trabalho, análise e interpretação dos dados e da revisão e aprovação da versão final deste artigo.

\section{CONFLITOS DE INTERESSE}

Os autores deste trabalho não possuem conflitos de interesse a serem declarados.

\section{AGRADECIMENTOS}

Ao Ministério da Educação (MEC) pelo financiamento (Edital PROEXT - 2014), às Pró-reitorias de Pesquisa e de Extensão da Universidade Federal de Juiz de Fora (UFJF) e à Residência Multiprofissional em Saúde do Adulto/HU-UFJF.

\section{REFERÊNCIAS}

1. World Health Organization. Obesity: prevention and managing the global epidemic. Report of a WHO Consultation. WHO Technical Report Series 894; 2000.

2. Instituto Brasileiro de Geografia e Estatística. Ministério do Planejamento, Orçamento e Gestão. Pesquisa de orçamentos familiares 2008-2009: antropometria e estado nutricional de crianças, adolescentes e adultos no Brasil. Rio de Janeiro, RJ: IBGE; 2010. $130 \mathrm{p}$

3. Pinheiro ARO, Freitas SFT, Corso ACT. Uma abordagem epidemiológica da obesidade. Rev Nutr. 2004;17(4):523-33.

4. Freitas S, Lopes CS, Coutinho W, Appolinario JC. Tradução e adaptação para o português da Escala de Compulsão Alimentar Periódica. Rev Bras Psiquiatr. 2001;23(4):215-20.

5. American Psychiatric Association. Diagnostic and statistical manual of mental disorders. 4th ed. Washington (DC): American Psychiatric Press; 1994

6. Inelmen EM, Toffanello ED, Enzi G, Gasparini G, Miotto F, Sergi G, et al. Predictors of dropout in overweight and obese outpatients. Int J Obes (Lond). 2005;29(1):122-8.

7. Toral N, Slater B. Abordagem do modelo transteórico no comportamento alimentar. Ciênc Saúde Coletiva. 2007;12(6):1641-50.

8. Prochaska J0, DiClemente CC, Norcross JC. In search of how people change. Applications to addictive behaviors. Am Psychol. 1992;47(9):1102-14.

9. DiClemente CC, Schlundt D, Gemmell L. Readiness and stages of change in addiction treatment. Am J Addict. 2004;13(2):103-19.

10. Greene GW, Rossi SR, Rossi JS, Velicer WF, Fava JL, Prochaska J0. Dietary applications of the stages of change model. J Am Diet Assoc. 1999;99(6):673-8.

11. Bittencourt SA, Santos PL, Oliveira MS. Motivação para mudança: análise fatorial da URICA para hábitos alimentares. Psico-USF. 2012;17(3):497-505.

12. Duarte ACG. Avaliação nutricional: aspectos clínicos e laboratoriais. São Paulo: Atheneu; 2007.

13. World Health Organization. Obesity: prevention and managing the global epidemic. Report of a WHO Consultation. WHO technical report series; 2000. 894p.

14. Bittencourt SA. Motivação para a mudança: adaptação e validação da Escala URICA (University of Rhode Island Change Assessment) para o comportamento de comer compulsivo. Porto Alegre: Pontifícia Universidade Católica do Rio Grande do Sul; 2009.

15. Koehnlein EA, Salado GA, Yamada AN. Adesão à reeducação alimentar para perda de peso: determinantes, resultados e a percepção do paciente. Rev Bras Nutr Clin. 2008;23(1):56-65.

16. Moraes CF, Oliveira MS. A entrevista motivacional em grupos de obesas no tratamento da compulsão alimentar periódica. Aletheia. 2011;35-36:154-67.

17. Cowan R, Britton PJ, Logue E, Smucker W, Milo L. The relationship among the transtheoretical model of behavioral change, psychological distress, and diet attitudes in obesity: implications for primary care intervention. J Clin Psychol Med Settings. 1995;2(3):249-67.

18. Wee CC, Davis RB, Phillips RS. Stage of readiness to control weight and adopt weight control behaviors in primary care. J Gen Intern Med. 2005;20(5):410-5.

19. Sbrocco T, Osborn R, Clark RD, Hsiao CW, Carter MM. Assessing the stages of change among African American women in a weight management program. J Black Psychol. 2012;38(1):81-103.

20. Teixeira PJ, Going SB, Sardinha LB, Lohman TG. A review of psychosocial pre-treatment predictors of weight control. Obes Rev. 2005;6(1):43-65.

21. Chimenti BM, Bruno MLM, Nakasato M, Isosaki M. Estudo sobre adesão: fatores intervenientes na dieta hipocalórica de coronariopatas internados em um hospital público de São Paulo. Rev Bras Nutr Clin. 2006;21(3):204-10. 
22. Palavras MA, Kaio GH, Mari JJ, Claudino AM. Uma revisão dos estudos latino-americanos sobre 0 transtorno da compulsão alimentar periódica. Rev Bras Psiquiatr. 2011;33(Supl 1):81-94.

23. Davis C, Carter JC. Compulsive overeating as an addiction disorder. A review of theory and evidence. Appetite. 2009:53(1):1-8.
24. Passos TCBM, Yazigi L, Claudino AM. Aspectos ideativos no transtorno da compulsão alimentar periódica: estudo com o Rorschach. Psico-USF. 2008;13(1):69-74.

25. Assis MAA, Nahas MV. Aspectos motivacionais em programas de mudança de comportamento alimentar. Rev Nutr. 1999;12(1):33-41.

26. Cochrane $G$. Role for a sense of self-worth in weight-loss treatments: helping patients develop self-efficacy. Can Fam Physician. 2008;54(4):543-7. 